\title{
The epidemiological scenario of feline sporotrichosis in Rio de Janeiro, State of Rio de Janeiro, Brazil
}

\author{
Sandro Antonio Pereira ${ }^{[1]}$, Isabella Dib Ferreira Gremião ${ }^{[1]}$, \\ Amanda Akemi Braga Kitada ${ }^{[1]}$, Jéssica Sepulveda Boechat ${ }^{[1]}$, \\ Paula Gonçalves Viana ${ }^{[1]}$ and Tânia Maria Pacheco Schubach ${ }^{[1]}$
}

[1]. Laboratório de Pesquisa Clínica em Dermatozoonoses em Animais Domésticos, Instituto de Pesquisa Clínica Evandro Chagas, Fundação Oswaldo Cruz, Rio de Janeiro, RJ.

\begin{abstract}
Introduction: Sporotrichosis is a mycosis affecting both humans and animals. Within the context of the ongoing sporotrichosis epidemic in the State of Rio de Janeiro, Brazil, sick cats plays an important role in the zoonotic transmission. The aim of this study was to update the number of feline cases diagnosed at the Fundação Oswaldo Cruz (2005-2011). Methods: The medical records of the cats followed were reviewed; the inclusion criterion was the isolation of Sporothrix spp. in culture. Results: In total, 2,301 feline cases were identified. Conclusions: These results should alert sanitary authorities to the difficulties associated with sporotrichosis control.
\end{abstract}

Keywords: Sporothrix spp. Sporotrichosis. Cat. Zoonosis. Epidemiology.

Sporotrichosis is an implantation mycosis caused by different species of the genus Sporothrix. The disease occurs worldwide but is endemic in Latin America, especially in the State of Rio de Janeiro, Brazil, where the first epidemic of zoonotic sporotrichosis was described at the end of the $1990 \mathrm{~s}^{1}$. The animal species most affected is the cat, which can exhibit a wide spectrum of clinical signs ranging from a single cutaneous lesion to a disseminated form ${ }^{2,3}$. Unlike the lesions observed in humans and dogs, cutaneous lesions in felines carry a high parasite burden, making the cat an important source of infection in the zoonotic transmission of this fungus ${ }^{1,3,4}$. In the past 14 years, transmission of $S$. schenckii from scratches, bites or contact with sick cats has been reported in Brazil ${ }^{1}$. Between 1998 and 2004, 1,503 cases of feline sporotrichosis were reported from different municipalities in the State of Rio de Janeiro ${ }^{5}$. From 1998 to 2009 , approximately 2,200 human cases were reported in the same region, emphasizing the importance of this mycosis as a public health problem ${ }^{1}$. The purpose of this study was to update the number of cases of feline sporotrichosis diagnosed at the Laboratório de Pesquisa Clínica em Dermatozoonoses em Animais Domésticos (Lapclin-Dermzoo)/Instituto de Pesquisa Clínica Evandro Chagas (IPEC)/Fundação Oswaldo Cruz (FIOCRUZ) from January 2005 to December 2011.

\footnotetext{
Address to: Dr. Sandro Antonio Pereira. Laboratório de Pesquisa Clínica em Dermatozoonoses em Animais Domésticos/IPEC/FIOCRUZ. Av. Brasil 4365, Manguinhos, 21040-900 Rio de Janeiro, RJ, Brasil.

Phone/fax: 5521 3865-9553

e-mail: sandro.pereira@ipec.fiocruz.br

Received 3 May 2013

Accepted 14 August 2013
}

The medical records of the cats followed at Lapclin-Dermzoo from January 2005 to December 2011 were reviewed; the inclusion criterion was the diagnosis of sporotrichosis confirmed by isolation of Sporothrix spp. in culture. The data collected from each cat were stored and processed using the Statistical Package for the Social Sciences 16.0 (SPSS Inc., Chicago, IL, USA). Simple frequencies for the categorical variables (origin, gender, breed, reproductive status, the distribution of cutaneous lesions and the presence of at least 2 cats per domicile) and measures of tendency for the quantitative variables (age and the duration of cutaneous lesions before the first clinical visit) were determined. The frequencies of clinical characteristics of the cohort of cats were described by univariate analysis.

In total, 2,301 cats with sporotrichosis were diagnosed at IPEC/FIOCRUZ. Due to the retrospective nature of this study, not all data were available for every animal. In this analysis, regarding the variables origin, gender, breed and reproductive status, respectively, 206, 46, 172 and 206 records were excluded because of incomplete data. All of the cats were from metropolitan regions of the State of Rio de Janeiro, mainly Rio de Janeiro municipality $(61.2 \% ; n=2,095)$. The majority were male $(66.4 \% ; n=2,255)$, mongrel $(89.3 \% ; n=2,129)$ and unneutered $(73.1 \% ; n=2,281)$. The median age of the cats was 2 years old. The median time between the appearance of cutaneous lesions and the initial clinical visit was 8 weeks, and $951(48.6 \% ; n=1,955)$ cats presented with cutaneous lesions in 3 or more non-adjacent places. In 1,288 $(63.6 \% ; n=2,025)$ feline cases, the presence of at least 2 cats per domicile was reported.

This study represents the largest series of sporotrichosis infections in animals diagnosed by fungal isolation. The number of cases reported here was higher than the number described 
during the first 6 years of disease reporting ${ }^{5}$. However, the profile of the feline population and the clinical characteristics reported in other studies conducted in the same region have not changed over the years ${ }^{2,6}$. These findings suggest that preventive measures, such as early spaying, should be used for young adult cats.

Despite the outbreak of sporotrichosis in Rio de Janeiro since the end of the 1990s, the median time from observation of cutaneous lesions by the owner to the initial assessment by the veterinarian remains delayed, as reported previously $y^{2,6}$. This delay may be one of the factors enhancing the infection of other animals and humans because early laboratory and clinical diagnosis of feline cases allows for the immediate establishment of treatment and preventive measures for this mycosis.

Sporotrichosis persists as a neglected disease and constitutes a formidable public health problem in Rio de Janeiro, even though more than 4,000 humans ${ }^{7}$ and 3,800 cats had been diagnosed at IPEC/FIOCRUZ by 2012. Sporotrichosis in cats has been registered in several countries, but nowhere in the world has there been a reported outbreak of animal sporotrichosis of this magnitude. Feline cases have been reported in other Brazilian states during the last 20 years, especially in Rio Grande do Sul ${ }^{8,9}$ and São Paulo ${ }^{10}$. However, the reported number in the 2 largest studies in these regions totaled 120 cats $^{9,10}$, which highlights the severity of the epidemiological situation occurring in Rio de Janeiro.

The numbers reported in the present study represent only those feline cases diagnosed at IPEC/FIOCRUZ, a reference center for the diagnosis and treatment of fungal diseases, thus accounting for the probable majority of cases. However, other public and private institutions located in the same region also perform sporotrichosis diagnosis but were not included in this study.

In Rio de Janeiro, the free provision of public veterinary services and antifungal drug supplies is currently restricted to IPEC/FIOCRUZ, which is unable to meet the demand for veterinary medical assistance for all cases involved in this epidemic. Unfortunately, even with free clinical and laboratory evaluations, treatment abandonment occurs with high frequency in feline sporotrichosis cases and may represent an obstacle to the control of this disease ${ }^{11}$.

In our study, all feline isolates were morphologically identified as $S$. schenckii at the time of diagnosis. For 15 of the isolates, it was possible to characterize the species by reconstructing their phylogenetic origin using the calmodulin locus, which resulted in Sporothrix brasiliensis in all cases ${ }^{12}$. Studies to characterize the species of feline isolates from this epidemic and to determine their susceptibility to antifungal drugs are being conducted to better understand the clinical and epidemiological aspects of this mycosis.
Currently, determining the scale of feline epizootic sporotrichosis is very difficult because reporting of this disease is not required. We believe sporotrichosis control can be achieved through basic educational measures that emphasize the responsible ownership of animals, programs to limit feline reproduction and effective action on the part of governmental institutions responsible for public health. Furthermore, updating the number of feline cases diagnosed at Lapclin-Dermzoo/IPEC/ FIOCRUZ alerts health professionals, researchers and sanitary authorities to the difficulties related to sporotrichosis control.

\section{CONFLICT OF INTEREST}

The authors declare that there is no conflict of interest.

\section{REFERENCES}

1. Barros MB, Almeida Paes R, Schubach AO. Sporothrix schenckii and Sporotrichosis. Clin Microbiol Rev 2011; 24:633-654.

2. Schubach TM, Schubach A, Okamoto T, Barros MB, Figueiredo FB, Cuzzi T, et al. Evaluation of an epidemic of sporotrichosis in cats: 347 cases (1998-2001). J Am Vet Med Assoc 2004; 224:1623-1629.

3. Pereira SA, Menezes RC, Gremião ID, Silva JN, Honse CO, Figueiredo $\mathrm{FB}$, et al. Sensitivity of cytopathological examination in the diagnosis of feline sporotrichosis. J Fel Med Surg 2011; 13:220-223.

4. Xavier MO, Bittencourt LR, Silva CM, Vieira RS, Pereira HP. Atypical presentation of sporotrichosis: report of three cases. Rev Soc Bras Med 2013; 46:116-118

5. Schubach A, Barros MB, Wanke B. Epidemic sporotrichosis. Curr Opin Infect Dis 2008; 21:129-133.

6. Pereira SA, Passos SR, Silva JN, Gremião ID, Figueiredo FB, Teixeira JL, et al. Response to azolic antifungal agents for treating feline sporotrichosis. Vet Rec 2010; 166:290-294.

7. Silva MB, Costa MM, Torres CC, Galhardo MC, Valle AC, Magalhães MA, et al. Esporotricose urbana: epidemia negligenciada no Rio de Janeiro, Brasil. Cad Saude Publica 2012; 28:1867-1880.

8. Nobre MO, Castro AP, Caetano D, Souza LL, Meireles MC, Ferreiro L. Recurrence of sporotrichosis in cats with zoonotic involvement. Rev Iberoam Micol 2001; 18:137-140.

9. Madrid IM, Mattei AS, Fernandes CG, Nobre MO, Meireles MA. Epidemiological findings and laboratory evaluation of sporotrichosis: a description of 103 cases in cats and dogs in southern Brazil. Mycopathol 2012; 173:265-273.

10. Rossi CN, Odaguiri J, Larsson CE. Retrospective assessment of the treatment of sporotrichosis in cats and dogs using itraconazole. Acta Sci Vet 2013; 41:1112.

11. Chaves AR, Campos MP, Barros MBL, Carmo CN, Gremião IDF, Pereira SA, et al. Treatment abandonment in feline sporotrichosis - study of 147 cases. Zoonoses Public Health 2013; 60:149-153.

12. Rodrigues AM, Teixeira MM, Hoog GS, Schubach TMP, Pereira SA, Fernandes GF, et al. Phylogenetic analysis reveals a high prevalence of Sporothrix brasiliensis in feline sporotrichosis outbreaks. PLoS Negl Trop Dis 2013; 7: e2281. 